EchoGéo

ECHOGEO- $4 \mid 2008$

Archéologie et Géographie

\title{
Entretien avec Stéphane Ghiotti
}

\section{Olivier Ninot}

\section{Q OpenEdition}

Journals

\section{Édition électronique}

URL : https://journals.openedition.org/echogeo/2463

DOI : $10.4000 /$ echogeo.2463

ISSN : 1963-1197

\section{Éditeur}

Pôle de recherche pour l'organisation et la diffusion de l'information géographique (CNRS UMR 8586)

\section{Référence électronique}

Olivier Ninot, «Entretien avec Stéphane Ghiotti », EchoGéo [En ligne], 4 | 2008, mis en ligne le 25 mars 2008, consulté le 01 août 2021. URL : http://journals.openedition.org/echogeo/2463 ; DOI : https:// doi.org/10.4000/echogeo.2463

Ce document a été généré automatiquement le 1 août 2021

EchoGéo est mis à disposition selon les termes de la licence Creative Commons Attribution - Pas d'Utilisation Commerciale - Pas de Modification 4.0 International (CC BY-NC-ND) 


\title{
Entretien avec Stéphane Ghiotti
}

\author{
Olivier Ninot
}

1 Cet ouvrage intervient dans une actualité marquée par des sécheresses et des inondations, par la cristallisation de conflits autour de l'usage de l'eau, par des pollutions et une pression accrue sur la ressource. Se posent les questions de comment et par qui est gérée la ressource eau, dans quel cadre territorial, et celle de la place de l'eau dans les politiques et programmes d'aménagement du territoire. Par des études de cas localisés, par l'analyse du jeu des acteurs et par l'exploitation de textes juridiques et d'archives, S. Ghiotti décrypte la gestion territoriale de l'eau en France et propose une analyse sur le temps long des relations entre l'eau et les territoires d'une part et entre développement des territoires et gestion de l'eau d'autre part.

En France, c'est le bassin versant qui est le cadre territorial de gestion de l'eau. A partir de ce constat, l'ouvrage suit deux lignes directrices. La première est un examen critique $\mathrm{du}$ bassin versant comme cadre territorial de gestion de l'eau; la seconde vise à montrer les liens entre approche territoriale de la gestion de l'eau et les mouvements de centralisation - décentralisation en France.

3 L'ouvrage est structuré en trois parties. Dans la première, l'auteur montre que la gestion territoriale de l'eau en France a été confinée à celle des bassins versants, et a conduit à l'adoption dans de ce cadre de gestion d'autres pays, notamment en Europe. La reconnaissance institutionnelle du bassin versant consacre l'association d'impératifs économiques et environnementaux à partir une double perception de l'eau comme ressource (renvoyant au concept de bassin hydrographique) et comme milieu (renvoyant au concept d'hydrosystème). Dans la deuxième partie, l'auteur retrace l'histoire du concept de bassin versant et de ses relations avec la décentralisation depuis une approche naturaliste à une approche fonctionnelle de gestion centrée sur la Région. Les relations entre gestion de l'eau et développement local sont abordées à travers une approche comparative de différents bassins versants. En toile de fond, c'est la question de la définition d'une ou de politiques territoriales de l'eau qui est posée. Dans la troisième partie, c'est la pertinence du bassin versant comme cadre territorial de gestion de l'eau qui est examinée au regard de la complexité territoriale (multiplicité des intervenants, des domaines de compétences et des niveaux 
d'intervention) : quelle est la place de la gestion de l'eau et quel rôle est dévolu au bassin versant? La question de fond est celle d'une bonne gouvernance de l'eau, dans un contexte où les impératifs du développement durable fixent les lignes directrices des politiques de gestion de l'eau : durabilité, globalité, participation, territorialisation.

4 Manipulant un corpus important et diversifié de textes législatifs, réglementaires ou procéduraux, l'auteur adopte une approche à la fois diachronique (XVI ${ }^{e}-\mathrm{XXI}^{\mathrm{e}}$ siècle) et multiscalaire justifiée par la multiplication dans le temps et dans l'espace du nombre d'acteurs et de cadres territoriaux de différents niveaux. Le matériau utilisé, original et fécond, conduit souvent l'auteur loin du « terrain » pratiqué par les géographes. Celuici apparaît malgré tout par l'intermédiaire des illustrations (une partie des 10 tableaux, 12 figures, 21 cartes), ainsi que dans les exemples apportés par les études de cas choisies dans les montagnes méditerranéennes, en Ardèche et dans les Hautes Alpes. L'eau marqueur identitaire, ressource multidimensionnelle, est également présentée comme un potentiel pour le développement et la (re)valorisation des territoires ruraux. L'évolution des modes de gestion, définis par celle des usages, y est à l'origine d'une complexification territoriale. Ainsi, au-delà des enjeux liés à la ressource, il ressort du travail de S. Ghiotti que la gestion territoriale de l'eau stimule les relations entre les collectivités territoriales au sein du processus de décentralisation et renvoie à quelques une de ses principales questions. Celle du rôle de l'Etat dans la gestion des conflits potentiels entre intérêt général et intérêts locaux ou spécificités locales. Celle de l'entité territoriale pertinente pour la gestion des biens communs.

Compte rendu d'entretien avec Stéphane Ghiotti

5 EchoGéo

Votre livre montre comment la gestion territorialisée de l'eau peut être à l'origine de solidarités territoriales ou à l'inverse de partitions; à l'origine de convergences politiques comme de renforcement de tensions. Selon vous, au regard des enjeux attachés à sa gestion, l'eau a-t-elle finalement à l'avenir plus vocation à être le support et le vecteur de valeurs positives ou à être de plus en plus l'objet d'âpres compétitions ?

6 S. Ghiotti

En forçant le trait, les enjeux de la gestion par bassin est d'arriver à répartir un volume d'eau entre utilisateurs, avec forcement des « gagnants » et des «perdants ». Il s'agit donc distribuer les coûts sociaux et territoriaux de la gestion...chez le voisin si possible avec dans bon nombre de cas des compensations, financières, politiques....Il s'agit certes d'aspects pouvant entraîner des tensions et des conflits dont la phase armée est le stade ultime. Les guerres de l'eau n'auront pas lieu. La littérature montre que la coopération l'emporte (bassins transfrontaliers notamment, ce qui n'empêche pas des systèmes de domination, amont/aval, aval/amont, rive droite/rive gauche...) sur les conflits. Si des conflits violents existent ils sont limités dans le temps et dans l'espace et motivés par d'autres actions (conquêtes territoriales notamment). A la différence du pétrole, l'eau est une ressource renouvelable.

7 EchoGéo

Quels points communs peut-on trouver entre gestion territorialisée de l'eau et gestion d'autres biens communs? En quoi le cas de l'eau est-il révélateur de l'intégration de l'environnement dans les enjeux de la gouvernance territoriale par exemple?

8 S. Ghiotti

Les points communs sont l'accès, le partage, les règles d'utilisation.... En ce qui concerne l'effet révélateur, c'est principalement le changement d'échelle (temps et 
espace) de la problématique, le changement de son objet et l'invitation des nouveaux acteurs avec des nouvelles règles de régulation par rapport au modèle " traditionnel " politique et administratif. Nouvelles échelles, nouveaux problèmes, nouveaux acteurs, nouvelles méthodes..., les cartes sont en partie redistribuées et chacun essaie d'avoir le meilleur jeu.

EchoGéo

Dans votre analyse des acteurs de la gestion territorialisée de l'eau, le secteur privé apparaît finalement assez peu. Aura-t-il un rôle plus important à jouer à l'avenir aux côtés d'acteurs plus nombreux et plus divers, collectivités territoriales de niveaux différents, associations d'usagers ou de consommateurs, etc. ?

10 S. Ghiotti

Il est vrai que ce n'est pas le centre de l'ouvrage. Pourtant ces acteurs sont présents dans le système (Comité de bassin, les SAGE qui accueil des usagers privés...). Lorsque je parle de recomposition des rapports villes-campagne pour la maitrise des ressources, certains syndicats d'alimentation en eau potable sont des acteurs incontournables. Pour la vente d'eau brute également, en témoigne l'exemple des Société d'aménagement régional (BRL, SCP...). Face aux enjeux financiers, politiques et environnementaux (DCE), ils sont des acteurs, historiques, parmi d'autres avec des atouts importants (technique, technologique, personnels qualifiés, des moyens financiers ainsi que des relais politiques). Il ne fait pas de doute que leur rôle ne va pas décroître !

11 EchoGéo

Vous expliquez que le bassin versant, territoire de projet, participe d'une complexification territoriale. En quoi cette complexification est porteuse de dynamiques de développement ou au contraire de lourdeurs voire de blocages dans les processus de prise de décision?

12 S. Ghiotti

Je suis plutôt partisan de la complexité territoriale plutôt que la recherche illusoire de cet optimum territorial. Les acteurs capables de jouer au mieux avec les projets, les échelles, les financeurs....sont ceux qui s'en sortent le mieux. Reste ensuite la question de la coordination et de la transparence. L'intérêt est donc de regarder comment se mènent les scènes de gouvernance et se régulent les oppositions.

13 EchoGéo

Vous expliquez qu'un changement important en matière de gestion territorialisée de l'eau est d'être passé de la gestion au projet : s'agit-il d'une simple évolution ou d'une petite révolution?

14 S. Ghiotti

Il s'agit à mon sens d'une évolution dictée par la conjonction de plusieurs processus, mondialisation, construction européenne, décentralisation, nouvelles demandes et pratiques sociales envers l'environnement, nouvelles mobilités...

15 EchoGéo

Vous avez récemment développé des recherches sur le thème de la gestion de l'eau et de ses territoires dans d'autres pays, au Liban notamment. Que retenir des expériences des pays que vous avez étudiés? Observez-vous d'un cas à l'autre, des grandes tendances se dessiner? 


\section{S. Ghiotti}

L'approche de cette problématique par une méthodologie comparative témoigne que la gestion par bassin est une construction sociale, politique et historique. Ces recherches montrent aussi que les enjeux démocratiques (prix de l'eau...) sont centraux dans la conduite des différentes réformes. On observe également que la transposition d'un modèle, réapproprié par les organismes internationaux et les leaders politiques nationaux et régionaux est souvent partielle et partiale.

17 Propos recueillis par Olivier Ninot

\section{AUTEUR}

\section{OLIVIER NINOT}

L'entretien porte sur le livre de Stéphane Ghiotti Les territoires de l'eau : gestion et développement en France publié aux éditions du CNRS, collection Espaces et Milieux. février 2007, $246 \mathrm{p}$. 